\title{
Laboratory studies evaluating the efficacy of a novel orally administered combination product containing sarolaner, moxidectin and pyrantel (Simparica Trio $^{\mathrm{TM}}$ ) for the treatment and control of flea infestations on dogs
}

Kristina Kryda ${ }^{1 *}$, Sean P. Mahabir ${ }^{1}$, Lori Carter ${ }^{2}$, William R. Everett ${ }^{3}$, David R. Young ${ }^{4}$, Leon Meyer ${ }^{5}$, Mirjan Thys ${ }^{6}$, Sara Chapin', Susan J. Holzmer ${ }^{1}$ and Csilla Becskei ${ }^{6}$

\begin{abstract}
Background: Five studies were conducted to evaluate a novel oral combination tablet containing sarolaner, moxidectin and pyrantel (Simparica Trio ${ }^{\mathrm{TM}}$ ), for efficacy against induced flea infestations, speed of kill and effects on flea reproduction on dogs.

Methods: Based on pre-treatment flea counts, dogs were randomly allocated to treatment with a single, oral dose of either placebo or Simparica Trio ${ }^{\mathrm{TM}}$ at the minimum label dose of $1.2 \mathrm{mg} / \mathrm{kg}$ sarolaner, $24 \mathrm{\mu g} / \mathrm{kg}$ moxidectin and $5 \mathrm{mg} /$ kg pyrantel (as pamoate salt) on Day 0. All dogs were infested with approximately 100 unfed, adult fleas (C. felis or C. canis) prior to treatment and weekly for 5 weeks post-treatment. In Studies 1, 2 and 3, the number of viable fleas were comb-counted at $24 \mathrm{~h}$ after treatment and after each weekly infestation; Study 2 also included groups treated with tablets containing sarolaner-alone $(1.2 \mathrm{mg} / \mathrm{kg})$, moxidectin-alone $(24 \mu \mathrm{g} / \mathrm{kg})$ or pyrantel-alone $(5 \mathrm{mg} / \mathrm{kg})$. In Study 4 , flea counts were conducted at 3, 4, 8 and $12 \mathrm{~h}$ after treatment and subsequent weekly infestations to establish speed of kill. In Study 5 (flea reproduction), dogs were housed in an enclosure designed to facilitate collection of flea eggs.

Results: Efficacy of Simparica Trio ${ }^{\mathrm{TM}}$ against C. felis was $\geq 99.7 \%$ and against C. canis was $100 \%$ at $24 \mathrm{~h}$ after treatment and after subsequent infestations for at least 35 days. Treatment with sarolaner-alone had similar efficacy to Simparica

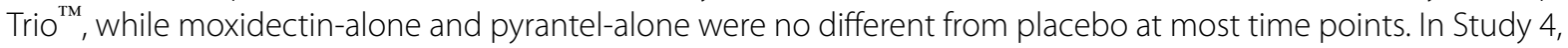
significant flea killing started at $4 \mathrm{~h}$ after treatment; by $8 \mathrm{~h}$ after treatment, all treated dogs were free of fleas. Following weekly re-infestation, the combination product reduced fleas by $\geq 97.8 \%$ within $12 \mathrm{~h}$ for 28 days. Simparica Trio ${ }^{\mathrm{TM}}$ reduced flea egg-laying by $100 \%$ for 35 days. No treatment-related adverse reactions occurred in any study.
\end{abstract}

Conclusions: A single dose of Simparica Trio $^{\text {TM }}$ at the recommended minimum dose provided highly efficacious and rapid treatment within $4 \mathrm{~h}$ of existing flea infestations and persistent control of fleas on dogs for 5 weeks. The efficacy against fleas resulted in 100\% prevention of flea reproduction for over a month following a single oral dose.

Keywords: Ctenocephalides canis, Ctenocephalides felis, Oral combination, Speed of kill

*Correspondence: kristina.a.kryda@zoetis.com

1 Zoetis, Veterinary Medicine Research and Development, 333 Portage

Street, Kalamazoo, MI 49007, USA

Full list of author information is available at the end of the article

c) The Author(s) 2020. This article is licensed under a Creative Commons Attribution 4.0 International License, which permits use, sharing, adaptation, distribution and reproduction in any medium or format, as long as you give appropriate credit to the original author(s) and the source, provide a link to the Creative Commons licence, and indicate if changes were made. The images or other third party material in this article are included in the article's Creative Commons licence, unless indicated otherwise in a credit line to the material. If material is not included in the article's Creative Commons licence and your intended use is not permitted by statutory regulation or exceeds the permitted use, you will need to obtain permission directly from the copyright holder. To view a copy of this licence, visit http://creativecommons.org/licenses/by/4.0/. The Creative Commons Public Domain Dedication waiver (http://creativecommons.org/publicdomain/zero/1.0/) applies to the data made available in this article, unless otherwise stated in a credit line to the data. 


\section{Background}

Dogs can be affected by numerous internal and external parasites which can have direct deleterious effects on their hosts and potentially transmit disease agents to dogs and humans $[1,2]$. Of the most common parasites, fleas frequently infest dogs worldwide [3] with the cat flea (Ctenocephalides felis felis) being the most common species followed by the dog flea (Ctenocephalides canis). Flea infestations are very common in dogs but often go unnoticed by pet owners [4] unless the dog develops pruritus or the flea infestation becomes severe, in which case newly emerged fleas may also jump on and bite the owner. Fleas cause irritation due to their direct bloodfeeding activity and heavy flea infestations may lead to anemia, especially in immature animals [5]. Dogs and cats can be highly sensitive to flea bites and the development of flea allergy dermatitis (FAD) is common [6, 7]. Fleas can transmit zoonotic pathogens such as Rickettsia felis [8], Rickettsia typhi [9] and Bartonella henselae $[10,11]$, and are the intermediate hosts for the tapeworm Dipylidium caninum [3]. Due to the ubiquitous nature of fleas, their ability to potentially induce clinical signs such as pruritus, and the possibility of transmitting diseases to the dog including zoonotic disease agents, efficacious treatment and control of flea infestations is a significant concern for pet owners and veterinarians [12]. To alleviate the direct negative impact of fleas and to reduce the risks of disease transmission, year-round flea control should therefore be considered for pets in most geographical areas $[3,13]$.

Sarolaner is an isoxazoline, a potent new class of ectoparasiticides in companion animals that provides broad activity against fleas and ticks [14]. Sarolaner inhibits the function of the neurotransmitter gamma aminobutyric acid (GABA) receptor and glutamate receptor, acting at the neuromuscular junction in insects which results in uncontrolled neuromuscular activity leading to death in fleas and ticks $[14,15]$. Sarolaner is rapidly absorbed following oral administration with $\mathrm{C}_{\max }$ occurring within the first day post dose and a half-life of 12 days [14]. Recently, a novel oral combination product that contains sarolaner in addition to moxidectin and pyrantel (Simparica Trio ${ }^{\mathrm{TM}}$, Zoetis, Parsipanny, NJ, USA) has been developed to provide not only treatment and control of flea and tick infestations for 1 month in dogs but also treatment of roundworm and hookworm infections and protection from heartworm and lungworm disease.

In this paper, we report a series of laboratory studies that evaluated the efficacy of Simparica Trio ${ }^{\mathrm{TM}}$ at a minimum label dose of $1.2 \mathrm{mg} / \mathrm{kg}$ sarolaner, $24 \mu \mathrm{g} / \mathrm{kg}$ moxidectin and $5 \mathrm{mg} / \mathrm{kg}$ pyrantel (as pamoate salt) against the most common flea species, C. felis and C. canis, infesting dogs. The studies include direct evaluation of treatment and prevention of flea infestations for 5 weeks after a single dose, flea speed of kill and effects on flea reproduction.

\section{Methods}

Five placebo-controlled, masked and randomized studies were conducted, including three dose confirmation studies. Studies 1 and 2 evaluated efficacy against the cat flea, C. felis. Study 2 included investigation of non-interference of the individual active components in the combination product and Study 3 measured efficacy against the dog flea, C. canis. Study 4 evaluated speed of kill against $C$. felis and Study 5 investigated the effects of the treatment on flea reproduction. The studies were conducted in accordance with the World Association for the Advancement of Veterinary Parasitology (WAAVP) guidelines for evaluating the efficacy of parasiticides for the treatment, prevention and control of flea and tick infestation on dogs and cats [16] and complied with Good Clinical Practice and VICH guideline GL9 [17]. Masking was accomplished by separation of functions of study personnel. All personnel conducting study observations were unaware of treatment assignments and dedicated personnel conducted the allocation of dogs and treatment dispensing but did not conduct any other study observations.

\section{Animals}

In Studies 1, 2, 3 and 4, 8 dogs were allocated per treatment group for each study in order to provide $>95 \%$ power to show both statistical significance and at least $90 \%$ reduction compared with placebo for each posttreatment flea count. This assumed a control mean flea count of 20-80 for the placebo. In Study 5, 10 dogs were allocated per treatment group in order to provide $>90 \%$ power to show both statistical significance and at least 90\% reduction compared to placebo for each flea egg count and number of larvae and adults emerged from incubated eggs. This assumed a control mean egg count of at least 100 for the placebo. Dogs were deemed to be in good health by a veterinarian at enrollment and had undergone a wash-out period determined sufficient by the investigator to ensure that no residual efficacy remained from any previously administered pulicidal compounds. Additionally, the adequacy of the washout period was confirmed via host suitability flea infestations and counts that were performed prior to study start which ensured that selected dogs could maintain an adequate infestation. Dogs were housed individually in enclosures that prevented any physical contact between animals and conformed to accepted animal welfare guidelines. For acclimatization, dogs were brought 
into the study site facilities for at least a week prior to treatment. Dogs were fed an appropriate maintenance amount of a commercial food and water was available $a d$ libitum for the duration of the study.

\section{Dose confirmation studies}

The studies used adult purpose-bred, Beagles and mixed breed dogs of both sexes, ranging in age from 6 months to 7 years and weighing from 6.1 to $25.0 \mathrm{~kg}$. Sixteen dogs were used in each of Studies 1 and 3, and 40 dogs were included in Study 2, as this non-interference study also included groups treated with individual components of the combination product.

\section{Speed of kill study}

Sixty-four Beagle and mixed breed dogs of both sexes, ranging in age from 10 months to 8 years and weighing from 6.5 to $15.7 \mathrm{~kg}$ were used in Study 4 .

\section{Flea reproduction study}

Twenty Beagles of both sexes, ranging in age from 3.6 to 4 years and weighing from 9.0 to $13.5 \mathrm{~kg}$ were used in Study 5.

\section{Design}

General health of all of the dogs was observed at least twice daily for the duration of the studies. To determine host suitability, dogs were infested with approximately 100 fleas prior to study start. The fleas were removed and counted $24 \mathrm{~h}$ after infestations. From a pool of dogs, dogs with the highest host suitability live flea counts were chosen for inclusion in each study. The dogs were blocked by host suitability flea counts and then assigned to treatment groups in a randomised complete block design.

\section{Dose confirmation studies}

In Studies 1 and 3, one placebo-treated group and one combination product-treated group were enrolled $(n=8$ per group). Study 2 included five treatment groups ( $n=8$ per group): placebo; combination product; sarolaneralone; moxidectin-alone; and pyrantel-alone.

\section{Speed of kill study}

Four separate pairs of placebo-treated and combination product-treated groups were used ( $n=8$ per group).

\section{Flea reproduction study}

One placebo-treated group and one combination product-treated group were enrolled ( $n=10$ per group).

\section{Treatment}

In Studies 1, 3, 4 and 5, dogs were dosed on Day 0 with either placebo tablets containing inert formulation ingredients (vehicle) or combination product tablets and in Study 2 additional groups were dosed with tablets containing the individual formulation components of the combination product (sarolaner-alone, moxidectin-alone or pyrantel-alone). Placebo and active tablets were similar in presentations to maintain masking. Tablets of varying strengths were provided, such that a combination of tablets could be administered to ensure dogs were appropriately dosed to the minimum end of the proposed label dose range. Each dog received a single or a combination of different tablet strengths containing all three active ingredients (or in Study 2 the individual formulation components) to provide as close as possible to the minimum label dosage of $1.2 \mathrm{mg} / \mathrm{kg}$ sarolaner (actual doses ranged from 1.2 to $1.6 \mathrm{mg} / \mathrm{kg}$ ), $24 \mu \mathrm{gg} / \mathrm{kg}$ moxidectin (actual doses ranged from 24 to $32 \mu \mathrm{g} / \mathrm{kg}$ ) and $5 \mathrm{mg} / \mathrm{kg}$ pyrantel (as pamoate salt) (actual doses ranged from 5.0 to $6.6 \mathrm{mg} / \mathrm{kg}$ ) or the equivalent number of placebo tablets based on pre-treatment body weights. Feed was withheld for at least $12 \mathrm{~h}$ prior to treatment and animals were not fed again until at least $4 \mathrm{~h}$ post-treatment. All doses were administered by hand pilling to ensure complete dosing. Each dog was observed for several minutes after dosing for evidence that the dose was swallowed and for up to $2 \mathrm{~h}$ post-dosing for any signs of emesis. Dogs were examined for general health and any reactions to treatment at $1,3,6$ and $24 \mathrm{~h}$ after treatment.

\section{Flea infestations}

Infestations were performed by applying the fleas directly to the fur while the dogs were restrained for a few minutes until the fleas dispersed into the hair coat. Approximately 100 unfed viable adult C. felis (C. canis in Study 3) were applied at each infestation to each dog.

\section{Dose confirmation studies}

Each dog was infested with fleas on Days-1, 6, 13, 20, 27 and 34. The fleas in Study 1 originated from a C. felis colony that had been initiated with fleas from Germany 6 years prior to the start of the study; new field-collected fleas from Ireland had been introduced to this colony 4 years prior to the study. The fleas in Study 2 originated from a $C$. felis colony that had been initiated with fleas from Kansas State University, Manhattan, Kansas, USA, to which additional fleas were periodically introduced from EL Labs, Soquel, California, USA, with the last introduction of new fleas occurring about 3 months prior to the study. The $C$. canis used in Study 3 originated from a colony that had been initiated with dog fleas collected from dogs in Ireland approximately 7 years prior to the study. 


\section{Speed of kill study}

The dogs were infested with fleas on Days-1, 7, 14, 21, 28 and 35 . The fleas originated from a $C$. felis colony which had been initially obtained from a laboratory colony in North Carolina, USA, enriched with wild caught fleas from Arkansas, USA, approximately 3 years prior to the study.

\section{Flea reproduction study}

The dogs were infested with fleas on Days-1, 5, 12, 19, 26 and 33. The fleas originated from a C. felis colony that had been initiated with fleas caught from naturally infested animals in California, USA. The colony was periodically enriched with locally-sourced wild fleas, with the last introduction occurring approximately 4 months prior to the study.

\section{Flea counts and evaluation of reproduction}

Flea counts were conducted by systematically combing the coat of each dog with a fine-toothed flea comb for at least $10 \mathrm{~min}$ to remove and count live fleas. Any dog on which fleas were found in the last $5 \mathrm{~min}(1 \mathrm{~min}$ in Studies 2 and 5) was combed for an additional $5 \mathrm{~min}(1 \mathrm{~min}$ in Studies 2 and 5) and this process was continued until no fleas were recovered during the final combing period. Protective clothing, flea combs and gloves were changed between animals.

\section{Dose confirmation studies}

Flea counts were conducted $24 \mathrm{~h}$ after treatment and after weekly re-infestations.

\section{Speed of kill study}

Flea counts were conducted $3,4,8$ or $12 \mathrm{~h}$ after treatment and after each weekly re-infestation for the four separate pairs of placebo-treated and combination producttreated groups.

\section{Flea reproduction study}

At $24 \mathrm{~h}$ after treatment and $48 \mathrm{~h}$ after each post-treatment re-infestation, each dog was held for $20 \mathrm{~h}$ in an enclosure specially designed to facilitate the collection of flea eggs. At the end of this period, adult flea counts were conducted on each dog and the fleas were removed from the dogs. For the flea egg collection, the animal's fur was ruffled by hand to dislodge any eggs retained in the coat and then all eggs were collected from the tray beneath the enclosure. For each dog, all eggs were counted and up to 100 randomly selected flea eggs were transferred to a container with an appropriate growth medium and maintained in an incubator under the appropriate conditions for egg hatching. After 5 days, the emerged viable larvae were counted. A further sample of up to 100 randomly selected flea eggs from each dog was transferred to a container with growth medium and maintained in an incubator under appropriate conditions for flea development, the emerged adult fleas being counted after 35 days.

\section{Statistical analysis}

Arithmetic means were used to summarize flea counts by treatment and day of study. Flea counts were analysed using a general linear mixed model for each day of study (SAS Release 9.4, SAS Institute Inc., Cary, North Carolina, USA). The model included the fixed effect of treatment and random effects of block and error. If multiple rooms were used the random effects included room, block within room, and error. Hypothesis testing was two-sided at the significance level of $\alpha=0.05$.

For adult flea, flea egg and flea larva counts, percent reduction relative to the placebo group (efficacy) was calculated using the formula: $[(\mathrm{C}-\mathrm{T}) / \mathrm{C}] \times 100$, where $\mathrm{C}$ is the mean count for the placebo group and $\mathrm{T}$ is the mean count for the treated group.

\section{Results}

\section{Dose confirmation studies}

Placebo-treated dogs consistently maintained flea infestations in all three dose confirmation studies. In the two studies with $C$. felis, arithmetic mean live flea counts for placebo-treated dogs ranged between 78.5-89.5 in Study 1 (Table 1) and 54.1-86.6 in Study 2 (Table 2). For C. canis mean counts for placebo dogs ranged between 74.8-87.9 in Study 3 (Table 1). Efficacy of the combination product against $C$. felis was $\geq 99.9 \%$ against existing infestations and at least $99.7 \%$ against subsequent infestations for 35 days after single treatment in both studies. For C. canis, efficacy was $100 \%$ against both existing infestations and against subsequent infestations for 35 days after a single treatment. Flea counts for the combination product were significantly lower $\left(7.78 \leq t_{d f} \leq 34.43,7 \leq d f \leq 35\right.$, $P<0.0001)$ than the placebo at all counts in all three studies (Tables 1 and 2).

In the non-interference evaluation (Study 2), treatment with sarolaner-alone resulted in $100 \%$ efficacy against existing infestations and efficacy of $97.6 \%$ or greater for at least 35 days after a single treatment. Flea counts for treated dogs were significantly lower $\left(7.78 \leq t_{(35)} \leq 10.42\right.$, $P<0.0001)$ than placebo at all counts (Table 2). Both moxidectin-alone and pyrantel-alone had little or no efficacy against fleas (Table 2). For moxidectin, flea counts were no different to those for placebo-treated dogs for all counts $\left(-1.81 \leq t_{(35)} \leq 1.49,0.0791 \leq P \leq 0.1442\right)$ with flea count reductions ranging from 0 to $15.2 \%$. Similarly, for pyrantel, flea counts were no different to those for placebo-treated dogs on most counts $\left(-1.30 \leq t_{(35)} \leq 0.57\right.$, $0.2018 \leq P \leq 0.6854$ ) with reductions ranging from 
Table 1 Arithmetic mean flea counts and percent efficacy relative to placebo at $24 \mathrm{~h}$ after treatment and weekly re-infestations with Ctenocephalides felis or C. canis for dogs treated orally with Simparica Trio ${ }^{\mathrm{TM}}$

\begin{tabular}{|c|c|c|c|c|c|}
\hline \multirow{2}{*}{$\begin{array}{l}\text { Day after } \\
\text { treatment }\end{array}$} & \multirow{2}{*}{$\begin{array}{l}\text { Treatment } \\
\text { group }\end{array}$} & \multicolumn{2}{|c|}{ Study 1 (C. felis) } & \multicolumn{2}{|c|}{ Study 3 (C. canis) } \\
\hline & & Flea count & $\%$ Efficacy & Flea count & \% Efficacy \\
\hline \multirow[t]{2}{*}{1} & Placebo & 89.5 & & 85.5 & \\
\hline & $\begin{array}{l}\text { Simparica } \\
\text { Trio }^{\text {TM }}\end{array}$ & $0.1^{*}$ & 99.9 & $0^{*}$ & 100 \\
\hline \multirow[t]{2}{*}{7} & Placebo & 78.5 & & 74.8 & \\
\hline & $\underset{\text { Trio }^{\mathrm{TM}}}{\text { Simparica }}$ & $0^{*}$ & 100 & $0^{*}$ & 100 \\
\hline \multirow[t]{2}{*}{14} & Placebo & 82.8 & & 75.3 & \\
\hline & $\begin{array}{l}\text { Simparica } \\
\text { Trio }^{\mathrm{TM}}\end{array}$ & $0^{*}$ & 100 & $0^{*}$ & 100 \\
\hline \multirow[t]{2}{*}{21} & Placebo & 80.5 & & 87.9 & \\
\hline & Trio $^{\text {Simparica }}$ & $0^{*}$ & 100 & $0^{*}$ & 100 \\
\hline \multirow[t]{2}{*}{28} & Placebo & 88.1 & & 81.8 & \\
\hline & $\begin{array}{c}\text { Simparica } \\
\text { Trio }^{\mathrm{TM}}\end{array}$ & $0^{*}$ & 100 & $0^{*}$ & 100 \\
\hline \multirow[t]{2}{*}{35} & Placebo & 89.5 & & 75.3 & \\
\hline & Trio $^{\text {SM }}{ }^{\text {Simparica }}$ & $0^{*}$ & 100 & $0^{*}$ & 100 \\
\hline
\end{tabular}

*Flea counts are significantly lower than placebo; $14.45 \leq t_{d f} \leq 34.43,7 \leq d f \leq 14$, $P<0.0001$

0 to $5.8 \%$. On Day 21, the flea count for the pyrantel group was significantly higher than that for placebo $\left(t_{(35)}=-3.00, P=0.005\right)$ and on Day 28, the flea count for pyrantel was significantly lower than that for placebo $\left(t_{(35)}=2.78, P=0.0087\right)$ with a reduction of $26.7 \%$.

\section{Speed of kill study}

In the speed of kill study (Study 4), all placebo-treated groups consistently maintained flea infestations for the duration of the study, with arithmetic mean counts between 72.4-98.8 fleas (Table 3). A significant reduction in flea counts compared to placebo $\left(t_{(53)}=4.16\right.$, $P=0.0001$ ) was first observed within $4 \mathrm{~h}$ after treatment and all treated dogs were free of fleas by $8 \mathrm{~h}$ after treatment. Following weekly re-infestation, the combination product significantly $\left(2.94 \leq t_{d f} \leq 19.46,10.6 \leq d f \leq 56\right.$, $P \leq 0.0138$ ) reduced flea counts relative to placebo within $4 \mathrm{~h}$ after infestations up to and including Day 21 and within $8 \mathrm{~h}$ through Day 35. At $12 \mathrm{~h}$ after post-treatment re-infestations, efficacy was $\geq 97.8 \%$ for 4 weeks and was $85.6 \%$ on Day 35.

\section{Flea reproduction study}

Placebo-treated dogs maintained flea infestations and fleas had good fecundity throughout Study 5.
Table 2 Arithmetic mean flea counts and percent efficacy relative to placebo at $24 \mathrm{~h}$ after treatment and weekly re-infestations with Ctenocephalides felis for dogs treated orally with Simparica Trio ${ }^{\mathrm{TM}}$, sarolaner-only, moxidectin-only or pyrantel-only tablets (Study 2)

\begin{tabular}{|c|c|c|c|}
\hline $\begin{array}{l}\text { Day after } \\
\text { treatment }\end{array}$ & Treatment group & Flea count & \% Efficacy \\
\hline \multirow[t]{5}{*}{1} & Placebo & 70.1 & - \\
\hline & Simparica Trio ${ }^{\mathrm{TM}}$ & $0^{*}$ & 100 \\
\hline & Sarolaner & $0^{*}$ & 100 \\
\hline & Moxidectin & 65.5 & 6.6 \\
\hline & Pyrantel & 66.6 & 5.0 \\
\hline \multirow[t]{5}{*}{7} & Placebo & 78.3 & - \\
\hline & Simparica Trio $^{\mathrm{TM}}$ & $0^{*}$ & 100 \\
\hline & Sarolaner & $1.9^{*}$ & 97.6 \\
\hline & Moxidectin & 66.4 & 15.2 \\
\hline & Pyrantel & 73.8 & 5.8 \\
\hline \multirow[t]{5}{*}{14} & Placebo & 65.8 & - \\
\hline & Simparica Trio ${ }^{\mathrm{TM}}$ & $0^{*}$ & 100 \\
\hline & Sarolaner & $0^{*}$ & 100 \\
\hline & Moxidectin & 70.6 & 0 \\
\hline & Pyrantel & 76.8 & 0 \\
\hline \multirow[t]{5}{*}{21} & Placebo & 54.1 & - \\
\hline & Simparica Trio $^{\mathrm{TM}}$ & $0.1^{*}$ & 99.8 \\
\hline & Sarolaner & $0.4^{*}$ & 99.3 \\
\hline & Moxidectin & 64.8 & 0.0 \\
\hline & Pyrantel & $71.8^{\mathrm{a}}$ & 0.0 \\
\hline \multirow[t]{5}{*}{28} & Placebo & 86.6 & - \\
\hline & Simparica Trio $^{\mathrm{TM}}$ & $0.3^{*}$ & 99.7 \\
\hline & Sarolaner & $0^{*}$ & 100 \\
\hline & Moxidectin & 78.9 & 8.9 \\
\hline & Pyrantel & $63.5^{\mathrm{b}}$ & 26.7 \\
\hline \multirow[t]{5}{*}{35} & Placebo & 79.6 & - \\
\hline & Simparica Trio ${ }^{\mathrm{TM}}$ & $0^{*}$ & 100 \\
\hline & Sarolaner & $0.4^{*}$ & 99.5 \\
\hline & Moxidectin & 79.4 & 0.3 \\
\hline & Pyrantel & 87.8 & 0 \\
\hline
\end{tabular}

*Flea counts are significantly lower than placebo; $7.78 \leq t_{(35)} \leq 10.42, P<0.0001$

a Flea count significantly higher than placebo; $t_{(35)}=-3.00, P=0.005$

b Flea count significantly lower than placebo; $t_{(35)}=2.78, P=0.0087$

Arithmetic mean live flea counts ranged between 60.8 73.8 and arithmetic mean flea egg counts were between 364.0-496.2 (Table 4). Arithmetic mean flea egg hatch rates for fleas from placebo-treated dogs ranged from $55.0 \%$ to $70.9 \%$ and $69.0 \%$ to $77.2 \%$ of eggs completed development to adult fleas. No live fleas were recovered and no flea eggs were collected from any dog treated with the combination product and thus there were no flea eggs available to evaluate for hatch or development to adult fleas. 
Table 3 Arithmetic mean flea counts (percent efficacy) relative to placebo for dogs treated orally with Simparica Trio ${ }^{\mathrm{TM}}$ at 3, 4, 8 or $12 \mathrm{~h}$ after treatment (Day 0) and weekly re-infestations with Ctenocephalides felis (Study 4)

\begin{tabular}{|c|c|c|c|c|c|c|c|}
\hline \multirow[t]{2}{*}{ Count time } & \multirow[t]{2}{*}{ Treatment group } & \multicolumn{6}{|c|}{ Day after treatment } \\
\hline & & 0 & 7 & 14 & 21 & 28 & 35 \\
\hline \multirow[t]{2}{*}{$3 \mathrm{~h}$} & Placebo & 77.1 & 95.0 & 89.0 & 93.8 & 90.4 & 98.8 \\
\hline & Simparica Trio $^{\mathrm{TM}}$ & $\begin{array}{l}77.8 \\
(0)\end{array}$ & $\begin{array}{l}61.6^{b} \\
(35.1)\end{array}$ & $\begin{array}{l}50.1^{b} \\
(43.7)\end{array}$ & $\begin{array}{l}88.3 \\
(5.9)\end{array}$ & $\begin{array}{l}92.8 \\
(0)\end{array}$ & $\begin{array}{l}93.0 \\
(5.8)\end{array}$ \\
\hline \multirow[t]{2}{*}{$4 \mathrm{~h}$} & Placebo & 72.4 & 94.1 & 89.5 & 93.1 & 95.9 & 97.4 \\
\hline & Simparica Trio ${ }^{\mathrm{TM}}$ & $\begin{array}{l}42.8^{\mathrm{a}} \\
(40.9)\end{array}$ & $\begin{array}{l}43.6^{b} \\
(53.7)\end{array}$ & $\begin{array}{l}31.3^{b} \\
(65.1)\end{array}$ & $\begin{array}{l}72.1^{b} \\
(22.6)\end{array}$ & $\begin{array}{l}85.9 \\
(10.4)\end{array}$ & $\begin{array}{l}95.9 \\
(1.5)\end{array}$ \\
\hline \multirow[t]{2}{*}{$8 \mathrm{~h}$} & Placebo & 84.0 & 88.9 & 89.1 & 87.8 & 94.0 & 94.1 \\
\hline & Simparica Trio $^{\mathrm{TM}}$ & $\begin{array}{l}0^{b} \\
(100)\end{array}$ & $\begin{array}{l}0.4^{b} \\
(99.6)\end{array}$ & $\begin{array}{l}6.9^{b} \\
(92.3)\end{array}$ & $\begin{array}{l}3.6^{b} \\
(95.9)\end{array}$ & $\begin{array}{l}26.6^{\mathrm{b}} \\
(71.7)\end{array}$ & $\begin{array}{l}70.4^{b} \\
(25.2)\end{array}$ \\
\hline \multirow[t]{2}{*}{$12 \mathrm{~h}$} & Placebo & 85.0 & 93.1 & 86.6 & 92.0 & 96.0 & 95.3 \\
\hline & Simparica Trio ${ }^{\mathrm{TM}}$ & $\begin{array}{l}0^{b} \\
(100)\end{array}$ & $\begin{array}{l}0^{b} \\
(100)\end{array}$ & $\begin{array}{l}0^{b} \\
(100)\end{array}$ & $\begin{array}{l}0^{b} \\
(100)\end{array}$ & $\begin{array}{l}2.1^{b} \\
(97.8)\end{array}$ & $\begin{array}{l}13.8^{b} \\
(85.6)\end{array}$ \\
\hline
\end{tabular}

a Mean live flea counts significantly lower than placebo; $t_{(53)}=4.16, P=0.0001$

b Mean live flea counts significantly lower than placebo; $10.09 \leq t_{d f} \leq 41.72,7 \leq d f \leq 56, P \leq 0.0138$

Table 4 Arithmetic mean flea and egg counts and percent efficacy relative to placebo after treatment and weekly re-infestations with Ctenocephalides felis for dogs treated orally with Simparica Trio $^{\mathrm{TM}}$ (Study 5)

\begin{tabular}{|c|c|c|c|c|c|}
\hline $\begin{array}{l}\text { Day after } \\
\text { treatment }\end{array}$ & $\begin{array}{l}\text { Treatment } \\
\text { group }\end{array}$ & Flea count & $\%$ Efficacy & Egg count & \% Efficacy \\
\hline \multirow[t]{2}{*}{1} & Placebo & 73.8 & & 364.0 & \\
\hline & Trio $^{\text {Simparica }}$ & $0^{*}$ & 100 & 0 & 100 \\
\hline \multirow[t]{2}{*}{7} & Placebo & 71.7 & & 444.5 & \\
\hline & $\begin{array}{l}\text { Simparica } \\
\text { Trio }^{\mathrm{TM}}\end{array}$ & $0^{*}$ & 100 & 0 & 100 \\
\hline \multirow[t]{2}{*}{14} & Placebo & 67.0 & & 496.2 & \\
\hline & $\underset{\text { Trio }^{\mathrm{TM}}}{\text { Simparica }}$ & $0^{*}$ & 100 & 0 & 100 \\
\hline \multirow[t]{2}{*}{21} & Placebo & 61.5 & & 488.5 & \\
\hline & $\underset{\text { Trio }^{\mathrm{TM}}}{\text { Simparica }}$ & $0^{*}$ & 100 & 0 & 100 \\
\hline \multirow[t]{2}{*}{28} & Placebo & 60.8 & & 440.6 & \\
\hline & $\begin{array}{l}\text { Simparica } \\
\text { Trio }^{\mathrm{TM}}\end{array}$ & $0^{*}$ & 100 & 0 & 100 \\
\hline \multirow[t]{2}{*}{35} & Placebo & 63.6 & & 366.8 & \\
\hline & $\begin{array}{c}\text { Simparica } \\
\text { Trio }^{\mathrm{TM}}\end{array}$ & $0^{*}$ & 100 & 0 & 100 \\
\hline
\end{tabular}

\section{Health observations}

No adverse events related to the treatment of the dogs with the combination product or single component oral tablets were noted in any of the studies. Two dogs in Study 4, one dosed with placebo the other with the combination product tablet, were noted to have a small amount of frothy yellow vomitus under their pens at 6 h post-dose. This was considered possibly related to the assisted administration of the tablets and resolved without treatment. The only other health observations were minor ailments such as dermatitis and otitis, typically expected in laboratory dogs exposed to repeated flea infestation.

\section{Discussion}

The studies reported here confirm the high efficacy of Simparica Trio ${ }^{\mathrm{TM}}$ against flea infestations for over a month after a single oral treatment at the minimum recommended dose. Efficacy was confirmed in three studies against the two most common flea species found on dogs, C. felis and C. canis, with flea counts for existing infestations reduced by $\geq 99.9 \%$ within $24 \mathrm{~h}$ after treatment, and $\geq 99.7 \%$ efficacy within $24 \mathrm{~h}$ against subsequent reinfestations for up to 5 weeks after treatment. In the noninterference study, comparison with the individual active ingredients of the combination tablet confirmed that flea efficacy was due to the sarolaner; the sarolaner-alone tablet had similar efficacy to the combination product at all time points, while the single component moxidectin and pyrantel tablets were no different to placebo at most time points $\left(-1.81 \leq t_{(35)} \leq 2.78,0.0791 \leq P \leq 0.6854\right)$. On Day 28 , the flea count for the single component pyrantel tablets was significantly lower by $26.7 \%$ than placebo $\left(t_{(35)}=2.78, P=0.0087\right)$ and on Day 21 it was significantly higher $\left(t_{(35)}=-3.00, P=0.005\right)$. The speed of kill study showed that treatment with the combination product resulted in the rapid onset of activity against fleas with live flea counts significantly reduced by $4 \mathrm{~h}$ after treatment. For post-treatment re-infestations, counts were significantly reduced from $3 \mathrm{~h}$ after infestation on Days 7 
and 14, from 4 hours on Day 21 and from 8 h on Days 28 and 35 . By $8 \mathrm{~h}$ after treatment efficacy was $100 \%$ and following re-infestation efficacy was $\geq 92.3 \%$ through Day $21,71.7 \%$ on Day 28 and $25.2 \%$ on Day 35 . By $12 \mathrm{~h}$ after treatment or re-infestation efficacy was $100 \%$ through Day $21,97.8 \%$ on Day 28 and $85.6 \%$ on Day 35.

A rapid speed of kill is an important characteristic of an effective pulicide, since it provides quick relief from the irritation of flea infestation which is especially critical for the management of FAD [18]. Additionally, providing efficacy early enough to break the life-cycle is critical to the control of infestations of the premises, killing fleas before they have a chance to lay eggs and re-infest households and pets [19]. The impact of the rapid speed of kill of Simparica Trio ${ }^{\mathrm{TM}}$ was demonstrated in the flea reproduction study where treatment resulted in the complete cessation of flea egg-laying, likely due to the direct efficacy against adult fleas. Thus, treatment completely halted the flea life-cycle.

Fleas are a year-round parasite of dogs throughout the world and regular parasiticidal treatment is needed to prevent infestations, ameliorate the irritation and debilitation of their blood-feeding, FAD, and reduce the potential risk for disease transmission [3]. Compliance by owners is best supported with simple, convenient, regular treatments that may be best achieved with combination broad-spectrum products. Flea control is best included in a management programme for the other major parasites of dogs that need regular treatment or prevention such as ticks, heartworm, lungworm and gastro-intestinal nematodes. Flea control with the combination product was rapid, highly effective, and persisted for at least a month following a single oral dose and also completely prevented flea reproduction. The combination of sarolaner, moxidectin and pyrantel in a single oral chewable tablet provides a convenient and highly effective monthly treatment that protects against fleas, as well as against other major parasites of dogs that can be given year-round.

\section{Conclusions}

A single oral dose of the combination product (Simparica Trio $^{\mathrm{TM}}$ ) administered at the recommended minimum label dose of $1.2 \mathrm{mg} / \mathrm{kg}$ sarolaner, $24 \mu \mathrm{g} / \mathrm{kg}$ moxidectin and $5 \mathrm{mg} / \mathrm{kg}$ pyrantel (as pamoate salt) was highly effective and provided rapid treatment of existing flea infestations and continuous control of fleas on dogs for a month. Both the cat flea (C. felis) and the dog flea (C. canis) were effectively controlled. Sarolaner was confirmed as the component providing the treatment and control of fleas. A single treatment started to kill the existing flea infestation within $4 \mathrm{~h}$ and subsequent re-infestations within $8 \mathrm{~h}$ for at least one month. Fleas were killed rapidly and resulted in the complete cessation of flea reproduction for over a month following a single oral dose.

\begin{abstract}
Abbreviations
FAD: Flea allergy dermatitis; GABA: Gamma aminobutyric acid; $\mathrm{VICH}$ : International Cooperation on Harmonization of Technical Requirements for Registration of Veterinary Medicinal Products; WAAVP: World Association for the Advancement of Veterinary Parasitology.
\end{abstract}

\section{Acknowledgements}

The authors would like to thank the participating personnel at the research laboratories for their assistance in conducting the studies. The authors would also like to thank Douglas Rugg for his assistance in preparing this manuscript.

\section{Authors' contributions}

$C B, K K$ and SPM conceived the study designs. KK drafted the manuscript. CB, DRY, KK, LC, LM, MT, NJ, SC, SJH and WE contributed to various aspects of conducting the studies. All authors were involved in protocol development, data interpretation, and manuscript review. All authors read and approved the final manuscript.

\section{Funding}

This study was funded by Zoetis, Parsippany, NJ, USA.

\section{Availability of data and materials}

Data upon which the conclusions are based have been presented in the article.

\section{Ethics approval and consent to participate}

Study protocols were reviewed and approved by the study site Institutional Animal Care and Use Committee or Ethical Review Committee prior to implementation.

\section{Consent for publication \\ Not applicable.}

\section{Competing interests}

This study was funded by Zoetis, Parsippany, NJ, USA. KK, SM, MT, SC, SJH and CB were employees of Zoetis. LC, WE, DRY and LM were contracted study investigators.

\section{Author details}

${ }^{1}$ Zoetis, Veterinary Medicine Research and Development, 333 Portage Street, Kalamazoo, MI 49007, USA. ${ }^{2}$ Stillmeadow, Inc., 12852 Park One Drive, Sugar Land, TX 77478, USA. ${ }^{3}$ BerTek, Inc., PO Box 606, Greenbrier, AR 72058, USA.

${ }^{4}$ Young Veterinary Research Services, Inc. (YVRS), 7243 East Ave, Turlock, CA 95380, USA. ${ }^{5}$ Clinvet International (Pty) Ltd, Uitsigweg, Bainsvlei, Bloemfontein 9338, Republic of South Africa. ${ }^{6}$ Zoetis, Veterinary Medicine Research and Development, Mercuriusstraat 20, 1930 Zaventem, Belgium.

Received: 1 July 2019 Accepted: 4 February 2020

Published online: 01 March 2020

\section{References}

1. Lee ACY, Schantz PM, Kazacos KR, Montgomery SP, Bowman DD. Epidemiologic and zoonotic aspects of ascarid infections in dogs and cats. Vet Parasitol. 2010;26:155-61.

2. Beugnet F, Marie J. Emerging arthropod-borne diseases of companion animals in Europe. Vet Parasitol. 2009;163:298-305.

3. Rust MK. The biology and ecology of cat fleas and advancements in their pest management: a review. Insects. 2017;8:118.

4. Farkas R, Gyurkovszky M, Solymosi N, Beugnet F. Prevalence of flea infestation in dogs and cats in Hungary combined with a survey of owner awareness. Med Vet Entomol. 2009;23:187-94.

5. Dryden MW. Host association: on-host longevity and egg production of Ctenocephalides felis. Vet Parasitol. 1989;34:117-22. 
6. Carlotti DN, Jacobs DE. Therapy, control and prevention of flea allergy dermatitis in dogs and cats. Vet Dermatol. 2000;11:83-98.

7. Blagburn BL, Dryden MW. Biology, treatment, and control of flea and tick infestations. Vet Clin Small Anim. 2009;39:1173-200.

8. Pérez-Osorio CE, Zavala-Velázquez JE, Arias León JJ, Zavala-Castro JE. Rickettsia felis as emergent global threat for humans. Emerg Infect Dis. 2008;14:1019-23.

9. Nogueras MM, Pons I, Pla J, Ortuño A, Miret J, Sanfeliu I, et al. The role of dogs in the eco-epidemiology of Rickettsia typhi, etiological agent of murine typhus. Vet Microbiol. 2013;163:97-102.

10. Chomel BB, Kasten RW, Floyd-Hawkins K, Chi B, Yamamoto K, RobertsWilson J, et al. Experimental transmission of Bartonella henselae by the cat flea. J Clin Microbiol. 1996;34:1952-6.

11. Breitschwerdt EB, Kordick DL. Bartonella infection in animals: carriership reservoir potential, pathogenicity, and zoonotic potential for human infection. Clin Microbiol Rev. 2000;13:428-38.

12. Beugnet F, Franc M. Insecticide and acaricide molecules and/or combinations to prevent pet infestation by ectoparasites. Trends Parasitol. 2012;28:267-79

13. Rust MK, Dryden MW. The biology, ecology, and management of the cat flea. Ann Rev Entomol. 1997;42:451-73.

14. McTier TL, Chubb N, Curtis MP, Hedges L, Inskeep GA, Knauer CS, et al. Discovery of sarolaner: a novel, orally administered, broad-spectrum isoxazoline ectoparasiticide for dogs. Vet Parasitol. 2016;222:3-11.
15. Garcia-Reynaga P, Zhao C, Sarpong R, Casida JE. New GABA/glutamate receptor target for [(3)H]isoxazoline insecticide. Chem Res Toxicol. 2013;26:514-6.

16. Marchiondo AA, Holdsworth PA, Fourie LJ, Rugg D, Hellmann K, Snyder $D E$, et al. World Association for the Advancement of Veterinary Parasitology (W.A.A.V.P.) second edition: guidelines for evaluating the efficacy of parasiticides for the treatment, prevention and control of flea and tick infestations on dogs and cats. Vet Parasitol. 2013;194:84-97.

17. European Medicines Agency. Guideline on good clinical practice. VICH Topic GL9. http://www.ema.europa.eu/docs/enGB/documentlibrary/ Scientificguideline/2009/10/WC500004343.pdf. Accessed 15 Apr 2019.

18. Rust MK. Advances in the control of Ctenocephalides felis felis (cat flea) on cats and dogs. Trends Parasitol. 2005;21:232-6.

19. Dryden MW. Flea and tick control in the 21 st century: challenges and opportunities. Vet Dermatol. 2009;20:435-40.

\section{Publisher's Note}

Springer Nature remains neutral with regard to jurisdictional claims in published maps and institutional affiliations.
Ready to submit your research? Choose BMC and benefit from:

- fast, convenient online submission

- thorough peer review by experienced researchers in your field

- rapid publication on acceptance

- support for research data, including large and complex data types

- gold Open Access which fosters wider collaboration and increased citations

- maximum visibility for your research: over 100M website views per year

At BMC, research is always in progress.

Learn more biomedcentral.com/submissions 